\title{
A new approach to obtain Kevlar-49 from PET waste bottles
}

\begin{abstract}
Non-biodegradable polyethylene terephthalate (PET) bottles have attracted increasing attention due to environmental concern in today's world. In order to reduce the amount of solid wastes generated and the dependency on fossil resources, a new approach has been conducted to prepare Kevlar-49 from PET waste bottles. Terephthalic acid, the main raw material used for preparation of Kevlar, was regenerated from PET waste bottles via subjection to a saponification process, whereas $\mathrm{p}$-phenylenediamine was prepared from PET waste bottles via the Hoffmann rearrangement method. Kevlar was synthesized from the reaction of terephthalic acid and p-phenylenediamine by polycondensation reaction. The structures of terephthalic acid, p-phenylenediamine and Kevlar were characterized by FT-IR, 1H NMR, 13C NMR, and elemental analysis (CHN). In this study, thermogravimetric analysis and differential scanning calorimetry, X-ray diffraction (XRD), as well as the mechanical properties (tensile strength, modulus, and percentage elongation at break) of the synthesized Kevlar-49, were compared with commercial Kevlar-49, prepared from the same raw materials, for better understanding of their properties.
\end{abstract}

Keyword: Poly(p-phenylene terephthalamide); Kevlar-49; PET waste bottles; Synthesis; New approach 